\title{
NOVAS CULTIVARES DE ACEROLA (Malpighia emarginata DC): UEL 3 - DOMINGA, UEL 4 - LÍGIA E UEL 5 - NATÁLIA ${ }^{1}$
}

\author{
VALÉRIA CARPENTIERI-PÍPOLO², CASSIOEGÍDIOCAVENAGHI PRETE², \\ MARIA GLÓRIA NILO GONZALEZ ${ }^{3}$, IRENE ODÍLIA POPPER ${ }^{4}$
}

\begin{abstract}
RESUMO - O programa de melhoramento genético de acerola, na Universidade Estadual de Londrina, iniciou-se em 1992, com a implatação de um pomar clonal constituído de genótipos selecionados no Norte do Paraná. As cultivares UEL 3-Dominga, UEL 4-Lígia, UEL 5-Natália são resultados de 5 ciclos de avaliações, onde foram considerados: precocidade, produtividade, conteúdo de vitamina C nos frutos, tolerância a pragas e doenças, tamanho e aparência dos frutos. As cultivares UEL 3-Dominga e UEL 5- Natália, destacamse pela produtividade das plantas, conteúdo de vitamina $\mathrm{C}$ e pelo tamanho do fruto. Em Londrina, a frutificação dessas cultivares concentra-se nos meses de novembro a março. A cultivar UEL 4-Lígia destaca-se pela precocidade e produtividade que, em Londrina, se concentra nos meses de outubro a março. A cultivar UEL 3-Dominga apresenta peso médio de frutos igual a 6,31g, Vitamina C igual a 2906 e $1250 \mathrm{mg} / 100 \mathrm{~g}$ em frutos verdes e maduros, respectivamente, acidez igual a $694,34 \mathrm{mg} / 100 \mathrm{~g}$ e Brix igual a $9,2^{\circ}$. A cultivar UEL 4Lígia apresenta peso médio dos frutos igual a 7,85g, vitamina C igual a 3579 e $1458 \mathrm{mg} / 100 \mathrm{~g}$ em frutos verdes e maduros, respectivamente, acidez igual a $1110 \mathrm{mg} / 100 \mathrm{~g}$ e Brix igual a 7,85. A cultivar UEL 5-Natália apresenta peso médio dos frutos igual a 7,47g, Vitamina C igual a 3134,5 e $1098 \mathrm{mg} / 100 \mathrm{~g}$ em frutos verdes e maduros, respectivamente, acidez igual a $725 \mathrm{mg} / 100 \mathrm{~g}$ e Brix igual a $7,6^{\circ}$. Estas três cultivares são indicadas para plantio no Norte do Estado do Paraná.
\end{abstract}

Termos para indexação: Malpighia spp., Cereja das Antilhas, melhoramento genético, cultivar.

\section{THREE NEW CARIBBEAN CHERRY (Malpighia emarginata DC) CULTIVARS: UEL-3 DOMINGA, UEL 4-LIGIA AND UEL 5-NATALIA}

\begin{abstract}
The Caribbean cherry breeding program at Londrina State University began in 1992 with the establishment of a clone orchard from selected genotypes collected in the north of Parana state. UEL 3 -Dominga, UEL 4-Lígia, UEL 5-Natália cultivars were selected after five assessment cycles taking in consideration earliness, yield, vitamin C fruit content, pest and disease tolerance, fruit size and appearance. UEL 3-Dominga and UEL 5-Natália were outstanding for plant yield, vitamin C content and fruit size. The fructification of these cultivars happens from November to March in Londrina. UEL 4-Lígia cultivar was outstanding for earliness and yield. Its fructification happens from October to March in Londrina. UEL 3-Dominga cultivar presented an average fruit weight of $6,31 \mathrm{~g}$, vitamin C content of 2.906 and $1.250 \mathrm{mg} / 100 \mathrm{~g}$ in unripe and ripe fruits respectively, acidity of $694.34 \mathrm{mg} / 100 \mathrm{~g}$ and Brix of $9,2^{\circ}$. UEL 4-Lígia cultivar presented an average fruit weight of 7,85g, vitamin C content of 3579 and $1.458 \mathrm{mg} / 100 \mathrm{~g}$ in unripe and ripe fruits respectively, acidity of $1110 \mathrm{mg} / 100 \mathrm{~g}$ and Brix of 7,85 . UEL 5-Natália cultivar presented an average fruit weight of 7,47g, vitamin C of $3.134,5$ and $1.098 \mathrm{mg} / 100 \mathrm{~g}$ in unripe and ripe fruits respectively, acidity of $725 \mathrm{mg} / 100 \mathrm{~g}$ and Brix of 7,6 $6^{\circ}$ These three cultivars are indicated for planting in Paraná state.
\end{abstract}

Index terms: Malpighia spp., Barbados Cherry, Plant Breeding, cultivar.

\section{INTRODUÇÃO}

A região Norte do Paraná é constituída por pequenas e médias propriedades rurais com área menor que 5 ha. Esta realidade foi preponderante para a definição de estratégias agrícolas para o produtor dessa região.

Frutas de importância econômica como a acerola (Malpighia emarginata D.C..) podem sevir como alternativa com boas perspectivas para o Estado, pois, além das condições favoráveis nos aspectos climatológicos, há a viabilização de utilização de áreas com relevo mais acidentado e a possibilidade de instalação na região Norte do Paraná, de pólos frutícolas.

A acerola tem despertado a atenção dos agricultores do
Estado do Paraná por seu elevado conteúdo de vitamina C (2.500 a $4.500 \mathrm{mg} / 100 \mathrm{~g}$ de polpa) em relação a outras frutíferas e por seu potencial para industrialização, uma vez que pode ser consumida sob forma de sucos, compotas, geléias, utilizada no enriquecimento de sucos e de alimentos dietéticos, na forma de alimentos nutracêuticos, como comprimidos ou cápsulas, empregados como suplemento alimentar, chás, bebidas para esportistas, barras nutritivas e iogurtes.

Este emergente e próspero segmento econômico da fruticultura, todavia, tem na inexistência de cultivares adaptadas um dos mais graves problemas, tornando urgente a realização de ações de pesquisas voltadas para sua solução.

A variabilidade genética existente em acerola é claramente

1 (Trabalho 218/2000). Recebido: 06/10/2000. Aceito para publicação: 12/11/2001.

2 Professor Associado. Depto de Agronomia, Universidade Estadual de Londrina. Londrina, PR , C.P. 6001, CEP 86051-990. E-mail: pipolo@uel.br

3 Professor Adjunto, Depto de Agronomia, Universidade Estadual de Londrina. Londrina, PR, C.P. 6001, CEP 86051-990.

4 Professor Adjunto, Depto de Tecnologia de Alimentos, Universidade Estadual de Londrina. Londrina, PR, C.P. 6001, CEP 86051-990. 
observada em pomares comerciais onde se encontram matrizes obtidas por sementes, com hábito de crescimento diferenciado e produção de frutos quantitativa e qualitativamente heterogênea. Esse fato causa um certo transtorno ao sistema de produção, pois dificulta a execução racional de todas as práticas culturais, desorganizando, principalmente, o sistema de comercialização da propriedade. A exploração dessa variabilidade pode permitir a identificação de genótipos superiores, portadores de características de interesse agronômico definidas (Neto, 1995).

Bezerra et al. (1992) avaliaram 14 clones de aceroleira quanto às características físicas e químicas dos frutos. Estes autores observaram que o peso dos frutos apresentou uma média geral de 4,0 gramas, o teor de sólidos solúveis totais variou de $6,4^{\circ}$ a $13,2^{\circ}$, a média de acidez foi em torno de $1,0 \%$ e os teores de ácido ascórbico variaram de 1.149,3 a 2.399,3 mg/100g.

Bosco et al. (1994) selecionaram 9 clones de aceroleira com base em características fenológicas da planta e morfológicas dos frutos. O rendimento de polpa foi superior a $90 \%$. O peso dos frutos variou de 7,02 g a 9,68 g; o diâmetro médio dos frutos foi $2,51 \mathrm{~cm}$ e, de forma geral, os clones selecionados apresentaram consistência, cor e sabor que atendem plenamente às exigências do mercado.

Menezes \& Alves (1994) avaliaram a qualidade póscolheita de frutos vermelhos e amarelos de acerola colhidos em um pomar comercial. As análises realizadas foram: teor de sólidos solúveis totais, acidez e vitamina C. Os autores relataram que não foram observadas diferenças significativas entre as acerolas vermelhas e amarelas com relação à qualidade dos frutos.

Para o IBRAF (1995), as indústrias de transformação recebem acerola com 7 a 7,5 brix, mais de $1.200 \mathrm{mg}$ de ácido áscórbico/100g de polpa, coloração alaranjada ou avermelhada e peso mínimo de 4,0 g. Em São Paulo, a CATI lançou a cultivar “ Waldy CATI 30", mensurada nas condições de Tietê- SP, apresentando frutos de coloração vermelho-intensa quando maduros, com pH de 3,48, 7,4 brix e teor de ácido ascórbico em frutos maduros de $1.493,29 \mathrm{mg} / 100 \mathrm{~g}$ de polpa (CATI,1997). Gonzaga Neto \& Bezerra (2000) selecionaram a cultivar de acerola Sertaneja. Esses autores relatam que, nas condições de Pernambuco, onde a frutificação ocorre durante todo o ano, esta cultivar apresenta rendimento de até $100 \mathrm{~kg} / \mathrm{planta} / \mathrm{ano}$, teor de vitamina $C$ superior a $1500 \mathrm{mg} / 100 \mathrm{~g}$ de polpa e com frutos resistentes após a colheita. Kanno et al. (2000) selecionaram, em pomar comercial em Junqueirópolis-SP, a cultivar Olivier, que apresenta moderada resistência a M. incognita, teor de ácido ascórbico de 2.178,8 mg/100g. de polpa em frutos verdes e 1567,2 em frutos maduros e brix de 9,92.

A presente pesquisa teve por objetivo descrever três novas cultivares de acerola selecionadas e adaptadas à região Norte do Paraná.

\section{MATERIAL E MÉTODOS}

O programa de melhoramento genético de acerola, na Universidade Estadual de Londrina, iniciou-se em 1992, com a implantação de um pomar de matrizes constituído de 31 genótipos derivados de estaquia de plantas selecionadas em pomares comerciais nos Estados do Paraná e São Paulo.

Utilizou-se delineamento experimental de blocos ao acaso, com três repetições. O espaçamento utilizado foi $4 \mathrm{~m}$ entre linhas e $3 \mathrm{~m}$ entre plantas, com 3 plantas, obtidas por estaquia, por repetição. A adubação foi realizada durante o plantio das mudas e não foram realizadas podas e adubação de reposição nas 5 primeiras safras.

Durante cinco safras, as plantas foram avaliadas quanto à adaptação ambiental, vigor, conformação da copa, produtividade efetiva, número de floradas e estabilidade da produção.

A caracterização do germoplasma foi realizada com base na relação de descritores mínimos para a acerola (OLIVEIRA et al., 1998 a,b) que são representados a seguir: 1 - conformação da copa; 2 - altura da planta; 3 - diâmetro da planta; 4 - ramificação; 5 - textura foliar; 6 - formação dos bordos da folha madura; 7 presença de pilosidade na folha; 8 - presença de pilosidade no ramo; 9 - tipo de florescimento; 10 - coloração dos lóbulos da corola das flores; 11 - coloração da casca do fruto imaturo; 12 coloração da casca do fruto maduro; 13 - coloração da polpa do fruto maduro; 14 - tamanho do fruto; 15 - consistência da polpa; 16 - sólidos solúveis determinado com refratômetro em amostras de frutos maduros (AOAC, 1970); $17-\operatorname{acidez}(\mathrm{ml} \mathrm{NaOH} \mathrm{1N/100g),}$ o teor de acidez para os 3 genótipos foi determinado a partir da neutralização da acidez da polpa da fruta por titulação com $\mathrm{NaOH}$ $0,1 \mathrm{~N} ; 18$ - ácido ascórbico (mg/100g), o teor de ácido ascórbico, para os 3 genótipos, foi determinado em polpa macerada com 2,6 diclorofenol-indofenol; 19 - início de produção; 20 - peso do fruto (g);21 - rendimento de polpa (\%) obtido por meio da relação entre peso da polpa e peso do fruto.

\section{RESULTADOS E DISCUSSÃO}

As cultivares UEL 3 - Dominga, UEL 4 - Lígia e UEL 5 Natália são resultado de seleção entre 31 genótipos introduzidos dos Estados de São Paulo e Paraná. As avaliações iniciaram-se em 1995. As cultivares selecionadas destacaram-se já naquele ano, tendo apresentado tolerância à geada ocorrida no mês de setembro.

As características das plantas e dos frutos das três cultivares são apresentadas na Tabela 1.

UEL 3 - Dominga destaca-se pela produtividade da planta, tamanho dos frutos e conteúdo de vitamina $\mathrm{C}$ igual a $2.906 \mathrm{e}$ $1.250 \mathrm{mg} / 100 \mathrm{~g}$ nos frutos verdes e maduros, respectivamente. A frutificação concentra-se nos meses de novembro a março, em Londrina-PR (Tabela 1).

UEL 4 - Lígia destaca-se pela precocidade e produtividade da planta. A frutificação concentra-se nos meses de outubro a março, em Londrina-PR. O conteúdo de vitamina C é igual a 3.579 e $1.458 \mathrm{mg} / 100 \mathrm{~g}$ em frutos maduros e verdes, respectivamente (Tabela 1).

UEL 5 - Natália destaca-se pela produtividade da planta, tamanho do fruto e conteúdo de vitamina $\mathrm{C}$ igual a 3.134,5 e 1.098 $\mathrm{mg} / 100 \mathrm{~g}$ nos frutos verdes e maduros, respectivamente. A frutificação concentra-se nos meses de novembro a março, em Londrina-PR (Tabela 1).

As cultivares UEL 3 - Dominga, UEL 4 - Lígia e UEL 5 Natália apresentaram, nas cinco safras de avaliação, média de 26 a $30 \mathrm{~kg} /$ planta/ano.

A frutificação em pomares de acerola varia conforme a eficiência das plantas na produção de pólen e na ação de agentes 
TABELA 1 - Caracterização dos acessos de acerola UEL 3 Dominga, UEL 4-Lígia e UEL 5-Natália. Londrina $-\mathrm{PR}, 1999$.

\begin{tabular}{|c|c|c|c|}
\hline \multirow[t]{2}{*}{ Caracteres* } & \multicolumn{3}{|c|}{ Acessos } \\
\hline & UEL 3 - Dominga & UEL 4 - Lígia & UEL 5 - Natália \\
\hline 1 & Globular & globular & globular \\
\hline 2 & 2,10 & 1,95 & 1,88 \\
\hline 3 & 3,00 & 2,85 & 2,92 \\
\hline 4 & Intermediária & intermediária & intermediária \\
\hline 5 & Coriácea & coriácea & coriácea \\
\hline 6 & Levemente ondulada & levemente ondulada & levemente ondulada \\
\hline 7 & Sim & $\operatorname{sim}$ & $\operatorname{sim}$ \\
\hline 8 & $\operatorname{Sim}$ & $\operatorname{sim}$ & $\mathrm{Sim}$ \\
\hline 9 & Flores agrupadas & flores agrupadas & flores agrupadas \\
\hline 10 & Róseo-escura & Róseo-escura & Róseo-escura \\
\hline 11 & Arroxeada & arroxeada & arroxeada \\
\hline 12 & Vermelha & vermelha & vermelha \\
\hline 13 & Vermelha & vermelha & vermelha \\
\hline 14 & Média & média & Média \\
\hline 15 & Firme & firme & Firme \\
\hline 16 & 9,2 & 7,2 & 7,6 \\
\hline 17 & 694,34 & 1110 & 725 \\
\hline 18 & maduras & maduras & maduros \\
\hline & 2.906 & 3.579 & $3.134,5$ \\
\hline 19 & outubro & setembro & outubro \\
\hline 20 & 6,31 & 7,85 & 7,47 \\
\hline 21 & $82,5 \%$ & $72,1 \%$ & $73,2 \%$ \\
\hline
\end{tabular}

*1 - conformação da copa; 2 - altura da planta (m); 3 - diâmetro da planta (cm); 4 - ramificação; 5 - textura foliar; 6 - formação dos bordos da folha madura; 7 - presença de pilosidade na folha; 8 - presença de pilosidade no ramo; 9 - tipo de florescimento; 10 - coloração dos lóbulos da corola das flores; 11 - coloração da casca do fruto imaturo; 12 - coloração da casca do fruto maduro; 13 - coloração da polpa do fruto maduro; 14 - tamanho do fruto; 15 - consistência da polpa; 16 - sólidos solúveis ( ${ }^{\circ}$ Brix); 17 acidez $(\mathrm{ml} \mathrm{NaOH} 1 \mathrm{~N} / 100 \mathrm{~g}) ; 18$ - ácido ascórbico $(\mathrm{mg} / 100 \mathrm{~g}) ; 19$ - início de produção; 20 - peso do fruto $(\mathrm{g}) ; 21$ - rendimento de polpa $(\%)$.

polinizadores. O pegamento dos frutos a partir de polinização cruzada é superior ao oriundo de autopolinização, e a polinização cruzada origina frutos de maior tamanho (Yamane \& Nakasome, 1961; Couceiro,1985; Magalhães et al 1999, ). Para assegurar uma boa dispersão de pólen e maior frutificação efetiva, recomendase, para as três cultivares, que o plantio seja realizado em filas intercalares ou intercalando-se plantas de outras cultivares de acerola com florescimento semelhante. Nunca se deve instalar o pomar com uma única cultivar.

As cultivares UEL 3-Dominga, UEL 4-Ligia e UEL 5-Natalia são sucetíveis aos nematóides de galhas $M$. javanica e $M$. incognita (Carpentieri-Pípolo et al., 2000).

\section{CONCLUSÃO}

Em razão da superioridade de suas características agronômicas, as cultivares UEL 3 - Dominga, UEL 4 - Lígia e UEL 5 - Natália são recomendadas para plantio no Norte do Paraná $\left(23^{\circ}\right.$ de latitude sul) ou em regiões de clima semelhante.

\section{REFERÊNCIAS BIBLIOGRÁFICAS}

AOAC. OFFICIAL METHODS OF ANALYSIS OF THE ASSOCIATION OF OFFICIAL ANALYTICAL CHEMISTS. 11. Ed. Washington 1970.105p.

BEZERRA, J. E. F.; LEDERMAN,I. E.; SILVA, M. F. F.; SOUZA, A. A. M. Enraizamento de estacas herbáceas de acerola com ácido alfanaftaleno acético a baixas concentrações em duas épocas. Revista Brasileira de Fruticultura, 1994, Salvador. Anais... p. 87.

BOSCO, J., FILHO, A. S. P.; NETO, M. B. Características fenológicas de plantas de aceroleira. CONGRESSO BRASILEIRO DE FRUTICULTURA, 1994. Salvador. Anais...p. 87.

CARPENTIERI-PÍPOLO,V.PRETE, C.E.C.; GONZÁLEZ,M.G.N.; POPPER,I.O.; BRUEL, D.C., DIAS,A M. Novas cultivares de Acerola :UEL 3-Dominga, UEL 4-Lígia, UEL 5-Natália. In: CONGRESSO BRASILEIRO DE FRUTICULTURA, 15., 1998, Poços de Caldas. Resumos... p.56.

CATI. CATI lança nova acerola: cultivar "Waldy" CATI 30. São Paulo, Governo do Estado de São Paulo, 1997. 3p.

COUCEIRO, E.M. Curso de extensão sobre a cultura da acerola. Recife: UFRPE, $1985.45 \mathrm{p}$.

GONZAGA NETO, L.; BEZERRA, J.E.F. Acerola Sertaneja In: Novas variedades Brasileiras de Frutas, Jaboticabal: Sociedade Brasileira de Fruticultura, 2000. p. 26-27.

INSTITUTO BRASILEIRO DE FRUTAS -IBRAF. Soluções fruta a fruta: Acerola. São Paulo,1995. 59p.

KANNO,O Y.; RIZZI,L.C.; KAVATI,R. Acerola Olivier. In: Novas variedades brasileiras de frutas, Jaboticabal: Sociedade Brasileira de Fruticultura, 2000. p. 24-25.

MAGALHÃES, L.M.; OLIVEIRA,D. DE; OHASHI, O. S. Efeito da polinização na frutificação da acerola. Revista Brasileira de Fruticultura, Jaboticabal, v.21, n1, p.95-97.1999

MENEZES, J. B., ALVES, R. E. Caracterização pós-colheita de acerolas vermelhas e amarelas colhidas em pomar comercial. CONGRESSO BRASILEIRO DE FRUTICULTURA, 1994, Salvador. Anais... p. 99.

NETO, G. L. Melhoramento genético da aceroleira. In: SÃO JOSÉ, A. R., ALVES, R. E. (ed.) Acerola no Brasil, produção e mercado. Vitória da Conquista-BA: UESB, 1995. 160p.

OLIVEIRA, J. R. P.; SOARES FILHO, W. dos S.; NASCIMENTO, A. S. do; COSTA, D. da C.; MATSURA, F. C. A. V.; GOMES, J. de C.; CARVALHO, J.E. B. de; REINAHRDT, D. H.; OLIVEIRA, R. P. Programa de pesquisa de acerola: Embrapa mandioca e fruticultura. Cruz das Almas, BA: Embrapa-CNPMF, 1998 a. 28 p. (Documento, 75).

OLIVEIRA, J. R. P.; SOARES FILHO, W. dos S.; CUNHA, R. B. da. Guia de descritores de acerola: versão preliminar. Cruz das Almas, BA: Embrapa-CNPMF, 1998 b. 22 p. (Documento, 84).

YAMANE,G.M.; NAKASOME, H.Y. Pollination and fruit set studies of acerola Malpighia glabra L. in Hawaii. Proceeding of the American Society for Horticultural Science, Geneva, v.28 p.141-148,1961. 